\title{
ON NORMAL SUBGROUPS OF DIRECT PRODUCTS
}

\author{
by F. E. A. JOHNSON
}

(Received 10th April 1989)

\begin{abstract}
We investigate the equivalence classes of normal subdirect products of a product of free groups $F_{n_{1}} \times \cdots \times F_{n_{k}}$ under the simultaneous equivalence relations of commensurability and conjugacy under the full automorphism group. By abelianisation, the problem is reduced to one in the representation theory of quivers of free abelian groups. We show there are infinitely many such classes when $k \geqq 3$, and list the finite number of classes when $k=2$.
\end{abstract}

1980 Mathematics subject classification (1985 Revision): $\quad 20$ E 07; 20 E 36.

\section{Introduction}

Two subgroups $H_{1}, H_{2}$ of a group $G$ are said to be conjugate in the generalised sense when $\alpha\left(H_{1}\right)=H_{2}$ for some automorphism $\alpha$ of $G$. Similarly, we may consider generalised commensurability, by which we mean that $\alpha\left(H_{1}\right) \cap H_{2}$ has finite index in both $\alpha\left(H_{1}\right)$ and $\mathrm{H}_{2}$. In this paper, we investigate the generalised conjugacy and commensurability classes of normal subgroups in a direct product of free groups; let $F_{k}$ denote the free group with basis $\left(X_{i}\right)_{1 \leqq i \leqq k}$, and for $0 \leqq d \leqq \min \{n, m\}$, let $N(n, m, d)$ be the subgroup of $F_{n} \times F_{m}$

$$
\text { generated by }\left\{\begin{array}{cc}
\left(X_{i}, X_{i}\right) & 1 \leqq i \leqq d \\
\left(X_{i}, 1\right) & d+1 \leqq i \leqq n \\
& \\
\left(1, X_{j}\right) & d+1 \leqq j \leqq m \\
\left(X_{i j}, 1\right) & 1 \leqq i<j \leqq d
\end{array}\right.
$$

where $X_{i j}$ is the commutator $X_{i j}=X_{i} X_{j} X_{i}^{-1} X_{j}^{-1}$. Then $N(n, m, d)$ is a finitely generated normal subgroup, with infinite index when $d \geqq 1$; it is, moreover, a subdirect product; that is, it projects epimorphically onto each factor.

We will show that, up to generalised commensurability, these are the only normal subdirect products.

Theorem A. Let $N$ be a normal subdirect product of $F_{n} \times F_{m}$ where $n, m \geqq 2$. Then there exists a unique integer $d$ with $0 \leqq d \leqq \min \{n, m\}$ such that, for some automorphism $\alpha$ of $F_{n} \times F_{m}, \alpha(N)$ has finite index in $N(n, m, d)$. 
By contrast, matters become more complicated when the number of factors exceeds three.

Theorem B. The set of generalised commensurability classes of normal subdirect products in $F_{n_{1}} \times \cdots \times F_{n_{k}}$ is infinite when $k \geqq 3$ and each $n_{i} \geqq 2$.

We note that the presence of the automorphism $\alpha$ in Theorem $A$ is essential; the set of commensurability classes is infinite when $k \geqq 2$ (see, for example, Remark (2.5) below). The number of generalised commensurability classes is also infinite for a product of two Surface groups [3].

Theorem $\mathrm{A}$ also allows us to give the following description of finitely generated normal subgroups of $F_{n_{1}} \times F_{n_{2}}$.

Corollary C. A nontrivial finitely generated normal subgroup $H$ of $F_{n_{1}} \times F_{n_{2}}$ has either finite index in one of the factors, or finite index in a subgroup isomorphic to $N\left(m_{1}, m_{2}, d\right)$, where $m_{r}=1+j_{r}\left(n_{r}-1\right)$, and $j_{r}$ is the index of the projection of $H$ in $F_{n_{r}}$.

The following consequence of Theorem A seems, despite its naturality, to have gone previously unremarked.

Corollary D. Let $G_{1}, \ldots, G_{k}$ be an arbitrary sequence of groups and let $H \triangleleft G_{1} \times \cdots \times$ $G_{k}$ be a normal subgroup. Then $H$ is finitely generated if and only if each $\pi_{i}(H)$ is finitely generated where $\pi_{i}: G_{1} \times \cdots \times G_{k} \rightarrow G_{i}$ is the projection onto the ith factor.

The author is indebted to his colleague. Dr A. H. Schofield for some invaluable discussions on the subject of "quivers".

\section{Product structures and abelianisation}

Let $G_{1}, \ldots, G_{k}$ be groups and let $\pi_{i}: G_{1} \times \cdots \times G_{k} \rightarrow G_{i}$ denote the $i$ th projection. We denote by $S\left(G_{1}, \ldots, G_{k}\right)$ the set of normal subdirect products of $G_{1} \times \cdots \times G_{k}$; that is

$$
S\left(G_{1}, \ldots, G_{k}\right)=\left\{\begin{array}{c}
N: N \text { is a normal subgroup of } \prod_{i=1}^{k} G_{i} \\
\text { and } \pi_{i}(N)=G_{i} \text { for each } i
\end{array}\right\} .
$$

The following proposition is elementary.

Proposition 1.1. Let $\phi_{i}: G_{i} \rightarrow H_{i}$ be surjective group homomorphisms for $1 \leqq i \leqq k$. If $N$ 
is a normal subdirect product of $H_{1} \times \cdots \times H_{k}$, then $\left(\phi_{1} \times \cdots \times \phi_{k}\right)^{-1}(N)$ is a normal subdirect product of $G_{1} \times \cdots \times G_{k}$.

In particular, when $\square_{i}: G_{i} \rightarrow G_{i}^{a b}$ is the abelianisation map, we get a function $\square^{-1}: S\left(G_{1}^{a b}, \ldots, G_{k}^{a b}\right) \rightarrow S\left(G_{1}, \ldots, G_{k}\right)$.

Proposition 1.2. For any groups $G_{1}, \ldots, G_{k}$, the map

$$
\square^{-1}: S\left(G_{1}^{a b}, \ldots, G_{k}^{a b}\right) \rightarrow S\left(G_{1}, \ldots, G_{k}\right) \text { is bijective. }
$$

Proof. Since $\square^{-1}$ is clearly injective, we show that it is also surjective. Write $G_{1} \times \cdots \times G_{k}$ as an "internal direct sum" $G=\tilde{G}_{1} \oplus \cdots \oplus \tilde{G}_{k}$, in which $\tilde{G}_{i}$ centralises $\tilde{G}_{j}$ for $i \neq j$. Then the commutator subgroup is also an internal direct sum $[G, G]=\left[\widetilde{G}_{1}, \tilde{G}_{1}\right] \oplus$ $\cdots \oplus\left[\tilde{G}_{k}, \tilde{G}_{k}\right]$. When $H$ is a normal subdirect product in $G$, we claim that each $\left[\tilde{G}_{i}, \tilde{G}_{i}\right] \subset H$. To see this, fix $i$ : let $x_{i}, y_{i} \in \widetilde{G}_{i}$, and choose $h \in H$ such that $\pi_{i}(h)=y_{i}$; that is, $h$ is a product $h=h_{1}, \ldots, h_{k}$ with $h_{j} \in \tilde{G}_{j}$ for $j \neq i$, and $h_{i}=y_{i}$.

Since $\tilde{G}_{i}$ centralises $\tilde{G}_{j}$ for $i \neq j$, and $H$ is normal in $G, x_{i} y_{j} x_{i}^{-1} y_{j}^{-1}=x_{i} h x_{i}^{-1} h^{-1} \in H$, so that $\left[\tilde{G}_{i}, \tilde{G}_{i}\right] \subset H$. Hence also $[G, G] \subset H$. Thus

$$
H=H[G, G]=\square^{-1} \square(H)
$$

so that $\square^{-1}$ is surjective, and hence also bijective.

Put $\operatorname{Aut}\left(G_{1}, \ldots, G_{k}\right)=\prod_{i=1}^{k} \operatorname{Aut}\left(G_{i}\right)$, considered as the group of product-preserving automorphisms of $G_{1} \times \cdots \times G_{k}$ in the obvious way. We consider the relation of conjugacy of subdirect products under Aut $\left(G_{1}, \ldots, G_{k}\right)$, strengthened by taking commensurability into account. Recall that two subgroups $A, B$ are commensurable when $A \cap B$ has finite index in both $A$ and $B$ : when $A$ and $B$ are normal subgroups, this is equivalent to saying that both $A$ and $B$ have finite index in $A B$. We obtain an equivalence relation ' $\approx$ ' on $S\left(G_{1}, \ldots, G_{k}\right)$ as follows;

$N_{1} \approx N_{2}$ if and only if $\alpha\left(N_{1}\right)$ is commensurable with $N_{2}$ for some $\alpha \in$ Aut $\left(G_{1}, \ldots, G_{k}\right)$; we write

$$
\mathscr{C}\left(G_{1}, \ldots, G_{k}\right)=S\left(G_{1}, \ldots, G_{k}\right) / \approx
$$

Let $F_{n}$ denote the free group of rank $n$. Abelianisation gives a map

$$
\square: F_{n_{1}} \times \cdots \times F_{n_{k}} \rightarrow \mathbf{Z}^{n_{1}} \oplus \cdots \oplus \mathbf{Z}^{n_{k}}
$$

and a bijection 


$$
\bigsqcup_{1}: S\left(F_{n_{1}}, \ldots, F_{n_{k}}\right) \rightarrow S\left(\mathbf{Z}^{n_{1}}, \ldots, Z^{n^{k}}\right)
$$

namely, the inverse of the map of (1.2). Let $\langle N\rangle$ denote the class of $N \in S\left(G_{1}, \ldots, G_{k}\right)$ in $\mathscr{C}\left(G_{1}, \ldots, G_{k}\right)$. If $\alpha \in \operatorname{Aut}\left(F_{n_{1}}, \ldots, F_{n_{k}}\right)$, let $\left.\alpha^{a b} \in \operatorname{Aut} \mathbf{Z}^{n_{1}}, \ldots, \mathbf{Z}^{n_{k}}\right)$ denote its abelianisation. If $H$ and $K$ are subgroups of $F_{n_{1}} \times \cdots \times F_{n_{k}}$, then

$$
\left[\square(H) ; \square(H) \cap \alpha^{a b} \square_{(K)}\right] \leqq[H ; H \cap \alpha(K)]
$$

so that the mapping $\Psi: \mathscr{C}\left(F_{n_{1}}, \ldots, F_{n_{k}}\right) \rightarrow \mathscr{C}\left(\mathbf{Z}^{n_{1}}, \ldots, \mathbf{Z}^{n_{k}}\right)$

$$
\Psi\langle N\rangle=\langle\square(N)\rangle
$$

is well defined.

Theorem 1.3. $\Psi: \mathscr{C}\left(F_{n_{1}}, \ldots, F_{n_{k}}\right) \rightarrow \mathscr{C}\left(\mathbf{Z}^{n_{1}}, \ldots, \mathbf{Z}^{n_{k}}\right)$ is bijective.

Proof. For $N \in \mathscr{C}\left(\mathbf{Z}^{n_{1}}, \ldots, \mathbf{Z}^{n_{k}}\right), \Psi \square^{-1}(N)=\langle N\rangle$, so that $\Psi$ is surjective. If $N_{1}, N_{2}$ are commensurable in $Z^{n_{1}} \oplus \cdots \oplus \mathbf{Z}^{n_{k}}$, then $\square^{-1}\left(N_{1}\right), \square^{-1}\left(N_{2}\right)$ are commensurable in $F_{n_{1}} \times \cdots \times F_{n_{k}}$; if $N_{1}$ is conjugate to $N_{2}$ under Aut $\left(Z^{n_{1}}, \ldots, Z^{n_{k}}\right)$, it follows from the Nielsen-Magnus Theorem $([7,8])$ on lifting automorphisms from $Z^{n}$ to $F_{n}$, that $\square^{-1}\left(N_{1}\right)$ is conjugate to $\square^{-1}\left(N_{2}\right)$ under $\operatorname{Aut}\left(F_{n_{1}}, \ldots, F_{n_{k}}\right)$. Thus $\Psi$ is injective.

\section{Subdirect products of abelian groups}

In this section, we consider generalised commensurability classes of subdirect products in $A_{1} \oplus \cdots \oplus A_{k}$, where $\left(A_{i}\right)_{1 \leqq i \leqq k}$ are finitely generated free abelian groups. We work in the category $A b\langle k\rangle$ of diagrams of homomorphisms of abelian groups over the diagram scheme $\langle *, k\rangle$.

$\langle *, k\rangle$ : the directed graph with vertex set $\{*, 1, \ldots, k)$, having arrows

$$
\phi_{r}: * \rightarrow r
$$

for $1 \leqq i \leqq k .\langle *, k\rangle$ is the dual of the " $k$-subspace quiver" $([2,4])$. Subdirect products $N$ of $A_{1} \oplus \cdots \oplus A_{k}$ may be considered as objects in $A b\langle k\rangle$ of the form

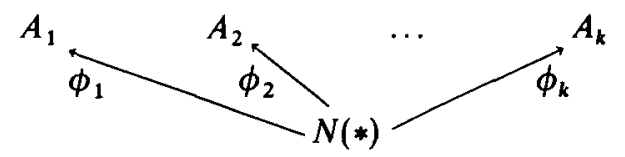

in which each $\phi_{r}$ is surjective, and the canonical morphism $N(*) \rightarrow \bigoplus_{i=1}^{k} A_{i}$ is injective. When $A_{1}, \ldots, A_{k}$ are understood, we confuse $N$ with $N(*)$. Such a diagram is said to be nondegenerate when $\bigcap_{i \neq j} \operatorname{Ker}\left(\phi_{j}\right)=0$ for each $i$. We write $\operatorname{Supp}(N)=\left\{j: A_{j} \neq 0\right\}$.

$A b\langle k\rangle$ has coproducts, defined by taking coproducts at each vertex. Similarly, one 
gets exact sequences in $A b\langle k\rangle$ by taking sequences which are exact at each vertex. Thus for subdirect products in particular, one obtains a coproduct pairing

$$
\square: S\left(A_{1}, \ldots, A_{k}\right) \times S\left(B_{1}, \ldots, B_{k}\right) \rightarrow S\left(A_{1} \oplus B_{1}, \ldots, A_{k} \oplus B_{k}\right)
$$

by means of $N \square M=\psi(N \oplus M)$ where

$$
\psi:\left(\bigoplus_{i=1}^{k} A_{i}\right) \oplus\left(\bigoplus_{i=1}^{k} B_{i}\right) \rightarrow \bigoplus_{i=1}^{k}\left(A_{i} \oplus B_{i}\right)
$$

is the obvious shuffling isomorphism.

An abelian subdirect product $N \in S\left(A_{1}, \ldots, A_{k}\right)$ gives rise to a canonical exact sequence as follows; let $N_{i}=N \cap A_{i}$, and let $N(i)$ be the subdirect product with $N(i)(*)=N_{i}=N(i)_{i}$, $\phi_{i}=I d$, and $N(i)_{j}=0$ for $i \neq j$; let $\tilde{N}$ be the subdirect product in which $\tilde{N}(*)=$ $N /\left(N_{1}+\cdots+N_{k}\right) ; \tilde{N}_{i}=A_{i} / N_{i} ;$ and where $\tilde{\phi}_{i}: \tilde{N}(*) \rightarrow \tilde{N}_{i}$ is the map induced from $\phi_{i}: N \rightarrow$ $A_{i}$. The following is clear.

Proposition 2.1. Each $N \in S\left(A_{1}, \ldots, A_{k}\right)$ decomposes functorially as an exact sequence $0 \rightarrow N(1) \square \cdots \square N(k) \rightarrow N \rightarrow \tilde{N} \rightarrow 0$ in which $\tilde{N}$ is nondegenerate, and $\operatorname{Supp}(N(i))=\{i\}$.

We define

$$
\begin{aligned}
& r_{i}(N)=r k_{Z}(N(i)(*)) \quad\left(=r k_{Z}\left(N_{i}\right)\right) \\
& d(N)=r k_{Z}(\tilde{N}(*)) \quad\left(=r k_{Z}\left(N /\left(N_{1}+\cdots+N_{k}\right)\right)\right.
\end{aligned}
$$

When $k=2$, the triple $\left(r_{1}, r_{2}, d\right)$ is a complete set of invariants for the generalised commensurability class of $N$; to clarify the statement, consider the following subdirect product diagrams:

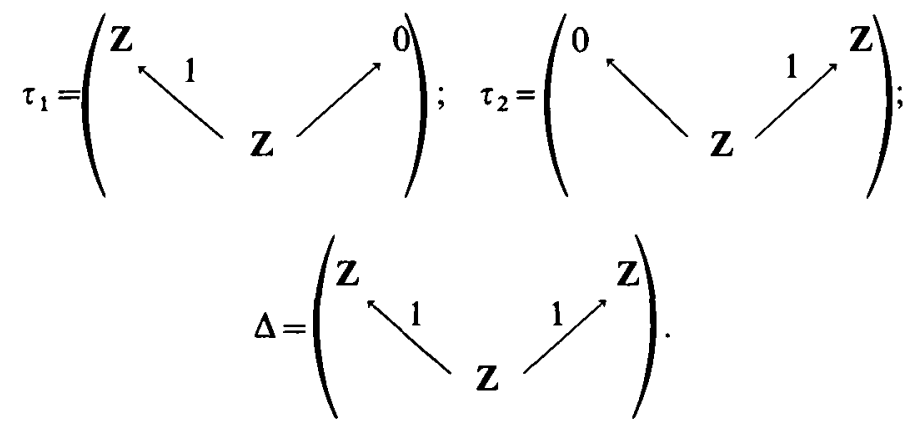

Theorem 2.2. Let $A_{1}, A_{2}$ be free abelian groups of finite rank, and let $N \subset A_{1} \oplus A_{2}$ be 
a subdirect product: if $N$ is maximal in its commensurability class then there is an isomorphism in $A b\langle 2\rangle$

$$
N \cong \tau_{1}^{r_{1}(N)} \square \tau_{2}^{r_{2}(N)} \square \Delta^{d(N)}
$$

Proof. The hypothesis that $N$ be maximal in its commensurability class is clearly equivalent to requiring that $\left(A_{1} \oplus A_{2}\right) / N$ be torsion free. Hence $\tilde{N}_{i}=A_{i} / N_{i}$, and $\tilde{N}(*)=N /\left(N_{1}+N_{2}\right)$ are free abelian. Moreover, $\tilde{\phi}_{i}: \tilde{N}(*) \rightarrow \tilde{N}_{i}$ is surjective; since $\tilde{N}$ is nondegenerate and $k=2$, each $\tilde{\phi}_{i}$ is also injective. (This fails for $k \geqq 3$.) Hence $\tilde{N}$ is a diagram of isomorphisms of free abelian groups;

$$
\tilde{N}=\left(\begin{array}{ccc}
\tilde{N}_{1} & & \\
& \phi_{1} & \\
& & \tilde{N}_{2} \\
& & \\
\phi_{2}
\end{array}\right)
$$

from which it follows immediately that $\tilde{N} \cong \Delta^{d(N)}$, since $d(N)=r k_{\mathrm{z}}(\tilde{N}(*))$.

Since $\tilde{N}$ is torsion free, we may find a complementary subgroup $\Delta(N)$ to $N_{1} \oplus N_{2}$ in $N ; N=N_{1} \oplus N_{2} \oplus \Delta(N)$. The restriction of each $\phi_{i}$ to $\Delta(N)$ is injective. Writing $C_{i}=\phi_{i}(\Delta(N))$, we see that $A_{i}=N_{i} \oplus C_{i}$, and that $N$ decomposes as a direct sum thus;

$$
N=N(1) \oplus N(2) \oplus \tilde{\Delta}
$$

where

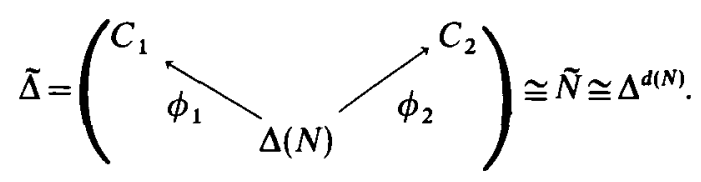

The result follows, since $N(i) \cong \tau_{i}^{r_{i}(N)}$.

If $N \subset A_{1} \oplus A_{2}$ is a subdirect product, $r k_{\mathrm{Z}}\left(A_{i}\right)=r_{i}(N)+d(N)$. We may rephrase things in the following way.

Corollary 2.3. If $N$ is a subdirect product of $\mathbf{Z}^{m} \oplus \mathbf{Z}^{n}$, its class in $\mathscr{C}\left(\mathbf{Z}^{m}, \mathbf{Z}^{n}\right)$ is completely specified by the single integer-valued invariant $d(N)$ which takes arbitrary values in the range $0 \leqq d(N) \leqq \min \{m, n)$; in particular, $\mathscr{C}\left(\mathbf{Z}^{m}, \mathbf{Z}^{n}\right)$ is finite.

By contrast, we have:

Proposition 2.4. If $k \geqq 3$, and each $n_{i} \geqq 1$, then $\mathscr{C}\left(Z^{n_{1}}, \ldots, Z^{n_{k}}\right)$ is infinite. 
Proof. Let $(a, b): \mathbf{Z}^{2} \rightarrow \mathbf{Z}$ be the mapping $(a, b)(x, y)=a x+b y$, where $a, b \in \mathbf{Z}$. For each integer $n \geqq 1$, the diagram

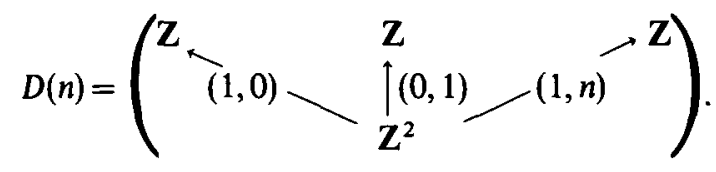

describes a nondegenerate subdirect product, maximal in its commensurability class, and an easy computation shows that $D(n)$ is not isomorphic to $D(m)$ in $A b\langle 3\rangle$ unless $n=m$. Thus $\mathscr{C}(\mathbf{Z}, \mathbf{Z}, \mathbf{Z})$ is infinite: by imbedding $\langle *, 3\rangle$ in $\langle *, k\rangle$, for $k \geqq 3$, and adding suitable degenerate summands, one sees that $\mathscr{C}\left(\mathbf{Z}^{n_{1}}, \ldots, \mathbf{Z}^{n_{k}}\right)$ is infinite for $k \geqq 3$.

Remark 2.5. Observe that the set of commensurability classes obtained from $S\left(\mathbf{Z}^{n_{1}}, \ldots, \mathbf{Z}^{n_{k}}\right)$ is always infinite, even for $k=2$ : for example, two maximal subgroups of rank 1 in $\mathbf{Z} \oplus \mathbf{Z}$ typically intersect in $\{0\}$; one obtains a corresponding statement for $S\left(F_{n_{1}}, \ldots, F_{n_{k}}\right)$ from (1.2). Thus the presence of the automorphism $\alpha$ in Theorem $\mathrm{A}$ is essential.

One may compare this with the analogous problem for finite dimensional rational vector spaces; then "commensurability" is the same as "identity". When $k \geqq 4,\langle *, k\rangle$ is not the Dynkin diagram of any simple Lie group, and $\mathscr{C}\left(\mathbb{Q}^{n_{1}}, \ldots, \mathbb{Q}^{n_{k}}\right)$ is infinite $[2,4]$. However, $\mathscr{C}\left(\mathbb{Q}^{n_{1}}, \ldots, \mathbb{Q}^{n_{k}}\right)$ is finite when $k \leqq 3 . \mathscr{C}\left(\mathbb{Q}^{n_{1}}, \mathbb{Q}^{n_{2}}\right)$ is described by a triple in a manner analogous to $\mathscr{C}\left(\mathbf{Z}^{n_{1}}, \mathbf{Z}^{n_{2}}\right)$, on replacing ' $r k_{\mathrm{Z}}$ ' by 'dim ${ }_{\mathbb{Q}}$ '; similarly $\mathscr{C}\left(\mathbb{Q}^{n_{1}}, \mathbb{Q}^{n_{2}}, \mathbb{Q}^{n_{3}}\right)$ is described by an 8-tuple, corresponding to the multiplicities of various indecomposable diagrams over $\langle *, 3\rangle$. We note, however, that $(-) \otimes_{\mathbf{Z}} \mathbb{Q}: \mathscr{C}\left(\mathbf{Z}^{n_{1}}, \ldots, \mathbf{Z}^{n_{k}}\right) \rightarrow \mathscr{C}\left(\mathbb{Q}^{n_{1}}, \ldots, \mathbb{Q}^{n_{k}}\right)$ fails to be injective for $k \geqq 3$.

\section{Normal subgroups of a direct product of free groups}

Our results so far depend for their expression on a particular product structure. In a direct product of free groups $F_{n_{1}} \times \cdots \times F_{n_{k}}$ with each $n_{k} \geqq 2$, however, the product structure is unique up to permutation of factors with the same rank ([5, Theorems 2.1, 2.7]), so for these groups, the notion of subdirect product is an intrinsic one. Indeed, the following is true (see, for example, [5, Corollary 2.9]).

Proposition 3.1. The group $\prod_{i=1}^{k} \operatorname{Aut}\left(F_{n_{i}}\right)$ of product-preserving automorphisms of $F_{n_{1}} \times \cdots \times F_{n_{k}}$ is a normal subgroup of finite index in the full automorphism group Aut $\left(F_{n_{1}} \times \cdots \times F_{n_{k}}\right)$ when each $n_{k} \geqq 2$.

Our results thereby assume an absolute character: 
Theorem 3.2. The set $\mathscr{G}\left(n_{1}, \ldots, n_{k}\right)$ of generalised commensurability classes of normal subdirect products of $F_{n_{1}} \times \cdots \times F_{n_{k}}$ is infinite when $k \geqq 3$ and finite when $k=2$, provided that each $n_{k} \geqq 2$.

Proof. Assume that each $n_{i} \geqq 2$. Then $\mathscr{G}\left(n_{1}, \ldots, n_{k}\right)$ is a quotient of $\mathscr{C}\left(F_{n_{1}}, \ldots, F_{n_{k}}\right)$ by the finite group

$$
\Phi=\operatorname{Aut}\left(F_{n_{1}} \times \cdots \times F_{n_{k}}\right) / \prod_{i=1}^{k} \operatorname{Aut}\left(F_{n_{i}}\right)
$$

However, by (1.3), (2.3) and (2.4), $\mathscr{C}\left(F_{n_{1}}, \ldots, F_{n_{k}}\right)$ is finite when $k=2$, and infinite when $k \geqq 3$, whence the result.

In the case of two factors, $F_{n} \times F_{m}$, we can give representatives for the generalised commensurability classes: we have bijections

$$
\mathscr{C}\left(F_{n}, F_{m}\right) \stackrel{\Psi}{\longrightarrow} \mathscr{C}\left(\mathbf{Z}^{n}, \mathbf{Z}^{m}\right) \stackrel{d}{\longrightarrow}\{0,1, \ldots, \min \{n, m\}\} .
$$

We define the diagonal rank $\delta(N)$ of a normal subdirect product, $N$, of $F_{n} \times F_{m}$ by

$$
\delta(N)=d(\Psi\langle N\rangle)
$$

All possible values of $\delta$ in the range $0 \leqq \delta \leqq \min \{n, m\}$ can occur. We may see this explicitly as follows; for $0 \leqq d \leqq \min \{n, m\}$, let $N(n, m, d)$ be the subgroup of $F_{n} \times F_{m}$ generated by:

$$
\begin{gathered}
\left(X_{i}, X_{i}\right)(1 \leqq i \leqq d) ;\left(X_{i}, 1\right)(d+1 \leqq i \leqq n) ;\left(1, X_{j}\right)(d+1 \leqq j \leqq m) ; \\
\left(X_{i j}, 1\right)(1 \leqq i<j \leqq d)
\end{gathered}
$$

where $\left\{X_{1}, \ldots, X_{k}\right\}$ denotes a free basis for $F_{k}$, and $X_{i j}$ denotes the commutator $X_{i j}=X_{i} X_{j} X_{i}^{-1} X_{j}^{-1}$.

Proposition 3.3. $N(n, m, d)$ is a normal subdirect product of $F_{n} \times F_{m}$ with $\delta(N(n, m, d)=d$.

Proof. Write $N=N(n, m, d) ; \xi_{i}=\eta_{\mathrm{i}}=\left(X_{i}, X_{i}\right) 1 \leqq i \leqq d$;

$$
\xi_{i}=\left(X_{i}, 1\right)(d+1 \leqq i \leqq n) ; \quad \eta_{i}=\left(1, X_{j}\right)(d+1 \leqq j \leqq m) ;
$$




$$
\xi^{i j}=\left(X_{i j}, 1\right)(1 \leqq i<j \leqq n) ; \quad \eta^{r s}=\left(1, X_{r s}\right)(1 \leqq r<s \leqq m)
$$

By definition, each $\xi_{i}, \eta_{i} \in N$, and it is easy to see that $\xi^{i j}, \eta^{r s} \in N$ for each $i, j, r, s$ with $1 \leqq i<j \leqq n$ and $1 \leqq r<s \leqq m$. If $W=W\left(X_{1}, \ldots, X_{k}\right)$ is a word in $\left\{X_{1}, \ldots, X_{k}\right\}$, let $w=w\left(\xi_{1}, \ldots, \xi_{k}\right)$ denote the corresponding word in $\left\{\xi_{1}, \ldots, \xi_{k}\right\}$. Then

$$
w \xi^{i j} w^{-1}=\left(W X_{i j} W^{-1}, 1\right)
$$

Since $\left[F_{n}, F_{n}\right]$ is generated by elements of the form $W X_{i j} W^{-1}$, we see that $\left[F_{n}, F_{n}\right] \times\{1\}$ $\subset N$. Replacing $\xi_{i}, \xi^{i j}$ by $\eta_{r}, \eta^{r s}$, we see, by symmetry, that $\{1\} \times\left[F_{m}, F_{m}\right] \subset N$. Hence $N=\square^{-1} \square(N)$, where

$$
\square: F_{n} \times F_{m} \rightarrow \mathbf{Z}^{n} \oplus \mathbf{Z}^{m}
$$

is the abelianisation map, so that $N$ is normal. $N$ is obviously a subdirect product, and $\delta(N)$ clearly takes the value $d$.

If $n \neq m, \operatorname{Aut}\left(F_{n}\right) \times \operatorname{Aut}\left(F_{m}\right)=\operatorname{Aut}\left(F_{n} \times F_{m}\right)$, whilst $\operatorname{Aut}\left(F_{n}\right) \times \operatorname{Aut}\left(F_{n}\right)$ has index 2 in $\operatorname{Aut}\left(F_{n} \times F_{n}\right)$, with the swap involution representing the nontrivial coset. Since each $N(n, n, d)$ is invariant under the swap, we obtain:

Theorem 3.4. If $2 \leqq m \leqq n, F_{n} \times F_{m}$ has precisely $m+1$ generalised commensurability classes of normal subdirect products: in particular, if $N$ is a normal subdirect product of $F_{n} \times F_{m}$, there exists a unique integer $d$ with $0 \leqq d \leqq m$ such that, for some automorphism $\alpha$ of $F_{n} \times F_{m}, \alpha(N)$ has finite index in $N(n, m, d)$.

Proof. Observe that $\bigsqcup_{(N(n, m, d))}$ is maximal in its commensurability class in $\mathbf{Z}^{n} \oplus \mathbf{Z}^{m}$, so that $N(n, m, d)$ is similarly maximal within $F_{n} \times F_{m}$. Note also that $N(n, m, d)$ is normal in $F_{n} \times F_{m}$. If $N$ is a normal subdirect product of $F_{n} \times F_{m}$ it follows from (1.3), (2.3) and (3.3), that one can find $\alpha \in \operatorname{Aut}\left(F_{n} \times F_{m}\right)$ such that $\alpha(N)$ and $N(n, m, d)$ are commensurable. By maximality of $N(n, m, d), \alpha(N)$ is contained with finite index in $N(n, m, d)$.

In general, a normal subgroup $H$ of $F_{n_{1}} \times F_{n 2}$ is a normal subdirect product of $\pi_{1}(H) \times \pi_{2}(H)$, where $\pi_{r}$ denotes projection onto the $r$ th factor; by the Nielsen-Schreier Theorem $([6$, p. 104$]), \pi_{r}(H)$ is either trivial or free; we obtain:

Corollary 3.5. A nontrival finitely generated normal subgroup $H$ of $F_{n_{1}} \times F_{n_{2}}$ has either finite index in one of the factors, or finite index in a subgroup isomorphic to $N\left(m_{1}, m_{2}, d\right)$, where $m_{r}=1+j_{r}\left(n_{r}-1\right)$, and $j_{r}$ is the index of the projection of $H$ in $F_{n_{r}}$. 
Finally, we observe:

Corollary 3.6. Let $G_{1}, \ldots, G_{k}$ be an arbitrary sequence of groups and let $H \triangleleft G_{1} \times \cdots \times$ $G_{k}$ be a normal subgroup. Then $H$ is finitely generated $\Leftrightarrow$ each $\pi_{i}(H)$ is finitely generated where $\pi_{i}: G_{1} \times \cdots \times G_{k} \rightarrow G_{i}$ is the projection onto the ith factor.

Proof. The implication " $\Rightarrow$ " is trivial. In proving " $\Leftarrow$ ", one may, by projection, reduce the problem to that for a subdirect product; that is, it suffices to prove the following for each $k \geqq 2$ :

$\mathscr{P}(k)$ : let $G_{1}, \ldots, G_{k}$ be finitely generated groups, and let $H$ be a normal subdirect product of $G_{1} \times \cdots \times G_{k}$. Then $H$ is finitely generated.

Suppose that $k=2$; in the special case where $G_{1}, G_{2}$ are free, the conclusion follows from (3.4), since $H$ is isomorphic to a subgroup of finite index in a group of the form $N(n, m, d)$, which is finitely generated by definition. In general, let $\phi_{i}: F_{i} \rightarrow G_{i}$ be an epimorphism from a finitely generated free group $F_{i}$. Then $\left(\phi_{1} \times \phi_{2}\right)^{-1}(H)$ is a normal subdirect product of $F_{1} \times F_{2}$ hence is finitely generated by the special case above. Thus $H$ is also finitely generated, being an epimorphic image of $\left(\phi_{1} \times \phi_{2}\right)^{-1}(H)$.

Suppose that $H$ is a normal subdirect product of $G_{1} \times \cdots \times G_{k+1}$. Let $K$ be the projection of $H$ in $G_{2} \times \cdots \times G_{k+1} ; K$ is a normal $k$-fold subdirect product, hence is finitely generated, by induction, and $H$ is a normal subdirect product of $G_{1} \times K$, so is finitely generated, by $\mathscr{P}(2)$.

The groups $N(n, m, d)$ are not finitely presented unless $d=0$. This follows easily from Theorem 2 of [1]. They also provide examples of Zariski dense, algebraically irreducible discrete subgroups of $S L_{2}(\mathbf{R}) \times S L_{2}(\mathbf{R})$, both of whose projections are discrete. Thus the simple picture provided by Borel's Density Theorem $([9, \mathrm{Ch} . \mathrm{V}])$ breaks down for Zariski dense subgroups of infinite covolume.

\section{REFERENCES}

1. G. Baumslag and J. E. Roseblade, Subgroups of direct products of free groups, London Math. Soc. (2), 30 (1984), 44-52.

2. I. N. Bernstein, I. M. Gelfand and V. A. Ponomarev, Coxeter functors and Gabriel's Theorem, Russian Math. Surveys 28 (1973), 17-32.

3. N. C. CARR, Subgroups of a product of surface groups, in preparation.

4. P. Gabriel, Unzerlegbare Darstellungen 1, Manuscripta Math. 6, (1973), 71-103.

5. F. E. A. Johnson, Automorphisms of direct products of groups and their geometric realisations, Math. Ann. 263 (1983), 343-364.

6. W. Magnus, A. Karrass and D. Solitar, Combinatorial group theory (Dover 1976).

7. W. MaGnus, Über n-dimensionale Gittertransformationen, Acta Math. 64 (1934), 353-367. 
8. J. Nielsen, Die Gruppe der dreidimensionalen Gittertransformationen, Kgl. Danske Videnskabernes Selbskab., Math. Fys. Meddelser V, 12, (1924), 1-29.

9. M. S. Raghunathan, Discrele subgroups of Lie groups Ergebnisse der Math. 68, SpringerVerlag 1972.

University COLLEGE LONDON

Gower Street

LONDON WCIE 6BT, UK 